\title{
Metabolic Engineering for Industrial Biotechnology
}

\section{Qingzhao Wang*}

Department of Microbiology and Cell Science, University of Florida, USA

Limited fossil resources and unstable supplies required the development of sustainable new ways to produce biochemicals and biofuels from renewable lignocelluloses, which was generally defined as industrial biotechnology [1]. This bioprocess usually included lignocelluloses hydrolysis by cellulase and the followed microbial fermentation. Compared to chemical process, industrial biotechnology may provide a low cost and low environment impact ways to produce important bulk chemicals and specialties.

People have long history of using microorganism fermentation to provide food and beverages. In last century, many important compounds were successfully fermented in industrial scale with the citric acid, antibiotic and amino acids as the representative. However, the old processes to develop these biocatalyst generally need long times and mostly depend on random mutagenesis. With the development and integration of modern molecular biology and applied engineering science, a new way of biocatalyst development was invented and it was called metabolic engineering. Metabolic engineering tried to improve production of metabolites and chemicals by rational design with the core technology of metabolic flux analysis and metabolic network analysis [2]. At this stage, metabolic engineering was largely applied in the carbon source utilization, transporter engineering, byproduct elimination and microbial cell properties improvements.

In this century, researchers felt that it is more and more difficult to further improve the strain because of our inability to decipher the systematic impacts of many different mutations. The recent breakthroughs in high-throughput techniques of genomics, transcriptomics, proteomics, metabolomics and fluxomics, which was defined in systems biology frameworks [3], had provided new opportunities for metabolic engineering. It was renamed by some researchers as systems metabolic engineering [4]. Together with computational tools, researchers can first time combine in silico genome scale metabolic networks analysis with other "omics" data to generate rational design of the metabolic modulation or whole metabolic pathways. This combination was especially promising for understanding of second metabolites metabolic control mechanisms and applying it for industrial production of specialties and biofuels.

As we know, the natural existing pathways may not be the best way to produce many target compounds and there are many target chemicals that cannot be produced from natural pathways either. To solve this problem, researchers created De novo pathways or synthetic pathways to better fulfill the mission. That was usually referred as synthetic biology [5]. This synthetic metabolic engineering had created many non-natural compounds and showed very good results in biofuel production.

Sometimes, we are still lacking possible ways to improve the target compounds titer and yield. At this time, metabolic evolution may be the good choice to employ. This strategy was based on natural selection and will generally select the desired phenotypes based on cell growth and ATP production. After the desired phenotypes were reached, the reversed metabolic engineering can be used to trace back the mutations for next round of engineering. The representative examples of metabolic evolutions are succinic acid producing Escherichia coli and D-lactate producing Bacillus coagulans [6,7].

Strategies for developing biocatalysts are now in transition from random mutagenesis and classic selection towards systems metabolic engineering and synthetic biology. However, it is still need to combine with some old strategy, such as metabolic evolution. Metabolic engineering is one of the fast developing research areas and will definitely contribute more for our new sustainable society in near future.

\section{References}

1. Papini M, Salazar M, Nielsen J (2010) Systems biology of industrial microorganisms. Adv Biochem Eng Biotechnol 120: 51-99.

2. Stephanopulos GN, Aristidou AA, Nielsen J (1998) Metabolic engineering: Principles and Methodologies. Academic press.

3. Wang QZ, Wu CY, Chen T, Chen X, Zhao XM (2006) Integrating metabolomics into a systems biology framework to exploit metabolic complexity: strategies and applications in microorganisms. Appl Microbiol Biotechnol 70: 151-161.

4. Lee JW, Na D, Park JM, Lee J, Choi S, et al. (2012) Systems metabolic engineering of microorganisms for natural and non-natural chemicals. Nat Chem Biol 8: 536-546.

5. Schwille P (2011) Bottom-up synthetic biology: engineering in a tinkerer's world. Science 333: 1252-1254.

6. Wang Q, Ingram LO, Shanmugam KT (2011) Evolution of D-lactate dehydrogenase activity from glycerol dehydrogenase and its utility for D-lactate production from lignocellulose. Proc Natl Acad Sci USA 108: 18920-18925.

7. Zhang X, Jantama K, Moore JC, Jarboe LR, Shanmugam KT, et al. (2009) Metabolic evolution of energy-conserving pathways for succinate production in Escherichia coli. Proc Natl Acad Sci USA 106: 20180-20185.

*Corresponding author: Qingzhao Wang, Department of Microbiology and Cell Science, University of Florida, Gainesville, FL 32611, USA, E-mail: wqz@ufl.edu

Received June 29, 2012; Accepted June 30, 2012; Published July 02, 2012

Citation: Wang Q (2012) Metabolic Engineering for Industrial Biotechnology. Single Cell Biol 1:e110. doi: 10.4172/2168-9431.1000e110

Copyright: (c) 2012 Wang Q. This is an open-access article distributed under the terms of the Creative Commons Attribution License, which permits unrestricted use, distribution, and reproduction in any medium, provided the original author and source are credited. 